\title{
Drug Repositioning: Auranofin as a Prospective Antimicrobial Agent for the Treatment of Severe Staphylococcal Infections.
}

\author{
Maria Iris Cassetta, ${ }^{[a]}$ Tiziano Marzo, ${ }^{[b]}$ Stefania Fallani, ${ }^{[a]}$ Andrea Novelli*[a] and Luigi Messori* ${ }^{[b]}$
}

\begin{abstract}
Auranofin, (AF), a gold(I) complex in clinical use for the therapy of rheumatoid arthritis, is reported here to produce remarkable bactericidal effects in vitro against Staphylococcus sp. Noticeably, a similar antimicrobial action and potency are also noticed toward a few MRSA strains but not toward E. Coli. The time and concentration dependencies of the antimicrobial actions of AF have been characterized through recording time kill curves, and a concentration dependent profile highlighted. Overall, the present results point out that auranofin might be quickly and successfully repurposed for the treatment of severe bacterial infections due to resistant Staphylococci.
\end{abstract}

Keywords: Auranofin • Antimicrobial drugs • Gold compounds • Drug repositioning $\bullet$ Staphyloccocal Infections

\section{Introduction}

Drug discovery and development is nowadays a very expensive, time-consuming, and risky process as witnessed by the limited number of new drugs approved every year. Attrition rates are extremely high, even during the late phases of clinical evaluation, with a huge financial impact. To speed up drug discovery and development and reduce failure rates and the associated costs, drug repositioning or repurposing may be considered as a highly effective and promising strategy (Chong et al. 2007, Aroson 2007).

Drug repositioning involves the investigation of drugs that were already approved for the treatment of other diseases and/or whose mechanisms or targets are known. Various methods including new screening platforms and advanced in silico and bioinformatic approaches may be exploited for the identification of the best candidates for drug repositioning. A few successful stories of drug repositioning are well documented in the recent literature such as those of finasteride, thalidomide, sildenafil and metformin (Cavalla 2013).

Auranofin, AF (I), is a mixed ligand gold compound in clinical use since 1985 for the treatment of severe rheumatoid arthritis (Ridaura) (Shaw 1999). It consists of a gold(I) center linearly coordinated to a triethylphosphine and a thiosugar ligand as shown in figure 1 .

[a] Dr. M.I. Cassetta, Dr. S. Fallani, Prof. A. Novell

Department of Healt Sciences

University of Florence

Viale Pieraccini 6, 50139 Florence (Italy)

E-mail: andrea.novelli@unifi.it

[b] Dr. T. Marzo, Prof. Luigi Messori

Department of Chemistry

University of Florence

Via della Lastruccia 3, 50019 Sesto Fiorentino (Italy)

E-mail: luigi.messori@unifi.it

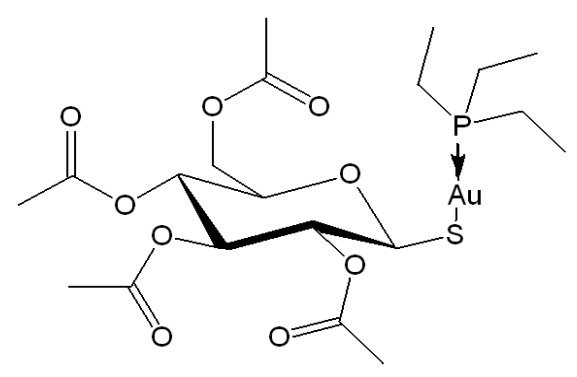

Figure 1. Chemical Structure of Auranofin

The presence of the strong phosphine ligand confers favourable pharmacological properties to this gold(I) center while the thiosugar ligand is a somewhat weaker ligand and may be released more easily. Following the release of the thiosugar, a coordination position is made available for gold(I) binding to biomolecules (Zou et al. 2000).

The development of AF as an antiarthritic drug was inspired by the traditional use of various gold compounds in the treatment of this disease dating back to the 1930s'. In comparison to other established antiarthritic gold compounds, AF has the big advantage of being administered orally. Yet, in spite of numerous investigations carried out so far, the actual mechanisms through which AF produces its favourable effects in RA are still unclear and largely unknown. There is just some limited evidence that $\mathrm{AF}$ can modulate the immune response and affect inflammation's mediators (Bondenson 1997, Messori et al 2004, Han et al. 2008).

Remarkably, auranofin combines the conspicuous affinity (after activation) of the gold(I) center for various "soft" donors (in particular $\mathrm{S}$ and $\mathrm{N}$ donors from proteins' side chains), with a moderate and largely acceptable systemic toxicity. This paves the way to its repositioning for new and different therapeutic uses. While the drug repositioning strategy has been extensively applied to several organic drugs, very limited examples still concern the case of inorganic drugs. Nevertheless, in recent years, a number of new attractive pharmaceutical actions were disclosed for AF including promising anticancer, antiviral and antiprotozoan properties;. in particular AF seems to be very effective for the treatment of of Schistosoma and also of Plasmodium falciparum infections (Shaw 1999, Kuntz 2007, Sanella et al. 2008). The state of art on the new medical uses of AF is described in detail in a comprehensive review article (Madeira et al. 2012).

Specifically, we wondered whether auranofin might possess effective antibacterial properties toward microorganisms that are difficult to treat with the available drugs and antibiotics. This is now a very urgent and unmet medical need as the number of innovative clinically approved antibiotics has dramatically decreased during the last two decades while an increasing number of multi resistant and highly dangerous bacteria (the so called superbugs) has appeared. Quite surprisingly, no systematic study exists in the literature on the antibacterial uses of AF; just some fragmentary observations are available (Novelli et al. 1999).

However, very recently, AF has been reported to be highly effective toward Clostridium difficile (Jackson-Rosario Self 2010), Treponema denticola (Jackson-Rosario Self 2009), and 
metronidazole-resistant Giardia lamblia (Tejman-Yarden et al. 2013) .

The above arguments led us to explore in more depth the antimicrobial properties of auranofin toward a few representative bacterial strains.

\section{Materials and methods}

Strains, antibiotics, and media

The strains used for the experiments were Escherichia coli ATCC 25922, Staphylococcus aureus ATCC 25923, Staphylococcus aureus USA 300, Staphylococcus epidermidis ATCC 12228, Staphylococcus epidermidis ATCC 35984 (biofilm producer) and 5 recent clinical isolates of methicillin-resistant Staphylococcus aureus (MRSA). Fresh dilutions of auranofin were prepared daily.

The in vitro experiments were performed in Mueller Hinton broth and Mueller Hinton agar (Oxoid, UK).

\section{MIC determination}

Minimal inhibitory concentrations (MIC) were determined according to the Clinical and Laboratory Standards Institute (CLSI) guidelines and using a broth dilution method. Briefly, two-fold broth serial dilutions were performed at inoculum sizes of $1 \times 10^{6} \mathrm{CFU} / \mathrm{ml}$ obtained from a $18-24 \mathrm{~h}$ incubation at $35^{\circ} \mathrm{C}$. Incubation of test tubes, containing a final volume of $1 \mathrm{ml}$, was done at $35^{\circ} \mathrm{C}$ for $18 \mathrm{~h}-24 \mathrm{~h}$. The MIC was defined as the lowest concentration of antimicrobial agent that inhibits the development of visible growth in the tubes (Clinical Laboratory Standards Institute 2004).

\section{Time-kill curves}

The killing activity of auranofin (I) and of its analogue (II) over time was evaluated in liquid medium (Mueller-Hinton Broth) at $37^{\circ} \mathrm{C}$ while shaking. The activity was examined against $S$. aureus ATCC 25923 at a final concentration of between $10^{6}$ colony forming units $(\mathrm{CFU}) / \mathrm{mL}$ and $10^{7} \mathrm{CFU} / \mathrm{mL}$ in either the presence or absence of auranofin (I) or derivative (II) at concentrations of 1/4xMIC, MIC, 2xMIC, 4xMIC, 16xMIC and 64xMIC. Bacterial growth was evaluated at time zero (before the addition of the two complexes) and at $1 \mathrm{~h}, 2 \mathrm{~h}, 4 \mathrm{~h}, 6 \mathrm{~h}$ and $24 \mathrm{~h}$ after addition of treatment agents, both in the control and antibiotic samples, using the $\mathrm{CFU} / \mathrm{mL}$ count method. At each hour, $0.1 \mathrm{~mL}$ of the sample removed from the bottles were diluted two-fold with normal saline $(0.9 \% \mathrm{NaCl})$ and spread on Mueller-Hinton agar plates using L-shaped rod and incubated for $24 \mathrm{~h}$ at $37^{\circ} \mathrm{C}$. Colony count of bacteria between $30-300 \mathrm{CFU} / \mathrm{mL}$ for each plate was determined to obtain time-kill curves (Clinical Laboratory Standards Institute 2004).

\section{Results and discussion}

AF was tested against two reference strains, namely Escherichia coli ATCC 25922 and Staphylococcus aureus ATCC 25923 that are representative of Gram negative and Gram positive bacteria, respectively. For comparison purposes parallel experiments were carried out on $\mathrm{AuClPEt}_{3}$ (II) an auranofin analogue where the thiosugar ligand is replaced by a chloride ligand (Shaw 1999).

The in vitro antimicrobial properties of both auranofin and its analogue were assessed by determination of the minimal inhibitory concentration (MIC) and by time-kill analysis. The high MIC values observed with both gold compounds in the case of E. coli ATCC 25922 strain $(>8 \mathrm{mg} / \mathrm{l})$ point out that both compounds are practically ineffective against Enterobacteriaceae. In contrast, both compounds are characterized by a remarkable in vitro activity with low MIC values (ranging from 0.125 to $0.5 \mathrm{mg} / \mathrm{l}$ ) against the $S$. aureus ATCC 25923 strain (Table 1).

\begin{tabular}{|l|c|c|}
\hline & $\begin{array}{c}\text { Auranofin } \\
(\mathbf{m g} / \mathbf{l})\end{array}$ & $\begin{array}{c}\text { AuCIP(Et) } \\
(\mathbf{m g} / \mathbf{l})\end{array}$ \\
\hline E. coli ATCC 25922 & $>8$ & $>8$ \\
\hline S. aureus ATCC 25923 & $0.25-0.5$ & $0.125-0.25$ \\
\hline S. aureus USA 300 & 0.25 & 0.125 \\
\hline S. aureus MRSA 1 & 0.25 & 0.125 \\
\hline S. aureus MRSA 2 & 0.25 & 0.125 \\
\hline S. aureus MRSA 3 & 0.25 & 0.125 \\
\hline S. aureus MRSA 5 & $0.125-0.25$ & 0.125 \\
\hline S. aureus MRSA 6 & 0.25 & 0.125 \\
\hline S. epidermidis ATCC 12228 & 0.25 & 0.125 \\
\hline $\begin{array}{l}\text { S. epidermidis ATCC 35984 } \\
\text { (biofilm } \text { producer) }\end{array}$ & 0.25 & 0.125 \\
\hline
\end{tabular}

Tab 1. MIC values for the two gold complexes Auranofin and $\mathrm{AuClP}(\mathrm{Et})_{3}$ against different bacteria strains (range from 4 experiments).

These observations prompted us to analyse in more detail the in vitro activity of $\mathbf{I}$ and II against different reference strains of the Staphylococcus genus including S. epidermidis and methycillin resistant S. aureus (MRSA) and also against recent clinical isolates of MRSA; indeed, this latter pathogen, causing extensive morbidity and mortality is considered worldwide as a very difficult one to treat and eradicate(Karampela et al. 2012). Interestingly, both gold compounds turned out to be very active in vitro against all tested strains, including all clinical isolates independently of being methycillin sensitive or resistant and biofilm producer or not. Typically, the resulting MIC values ranged from 0.125 to $0.5 \mathrm{mg} / \mathrm{l}$ and from 0.125 to $0.25 \mathrm{mg} / \mathrm{l}$, for compound I or II, respectively, with no significant differences on their in vitro antimicrobial potency (Table 1).

Time kill curves offer reliable and detailed information on the mode through which antimicrobial agents cause bacteria's death. The results obtained with the time-kill analysis in the case of $\mathbf{I}$ and II are shown in fig 2 and 3. Notably, such a method may give specific insight on the pharmaco-dynamic properties of the different drugs regarding either their concentration- or time-dependent activity (Craig 1998).

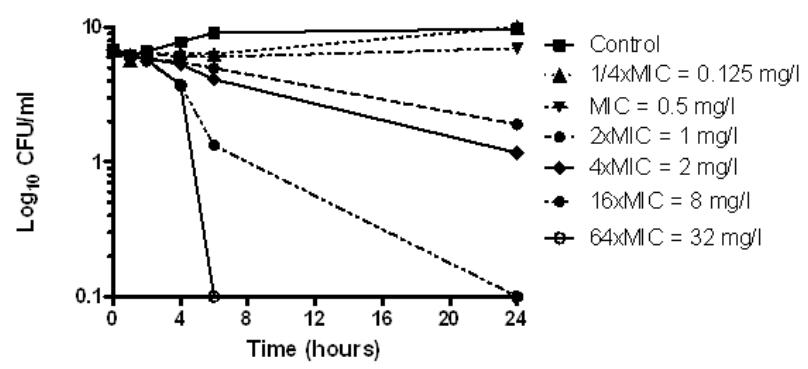

Figure 2. Killing curves with Auranofin against S. aureus ATCC 25923 (mean of two experiments) 


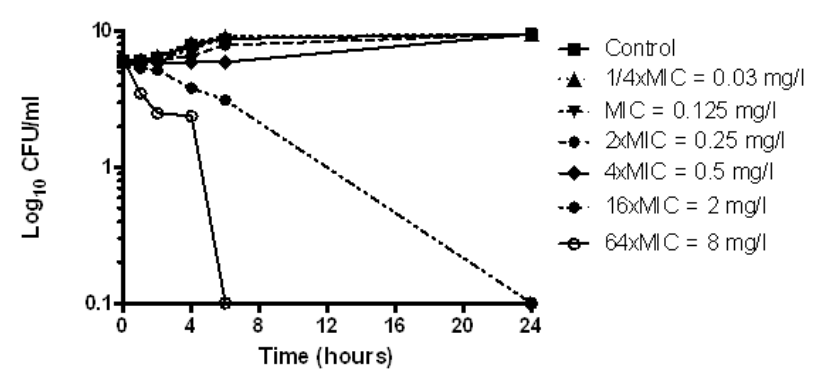

Figure 3. Killing curves with derivative (II) against S. aureus ATCC 25923 (mean of two experiments).

Analysis of the obtained killing curves indicates that the antibacterial activities of both auranofin and its analogue II on the $S$. aureus ATCC 25923 strain (with a MIC of $0.5 \mathrm{mg} / \mathrm{L}$ and 0.125 for I and II respectively) are very pronounced. Auranofin showed inhibition of bacterial growth for $12 \mathrm{~h}$ at MIC and for $24 \mathrm{~h}$ at $2 \times \mathrm{MIC}$, whereas no re-growth was observed even after $24 \mathrm{~h}$ at 16 and $64 \times$ MIC (fig. 2). Compound II showed a somewhat lower antibacterial profile with inhibition of bacterial growth for $8 \mathrm{~h}$ at $4 \times$ MIC, though no regrowth was observed after $24 \mathrm{~h}$ at 16 and $64 \times$ MIC (fig. 3). Auranofin demonstrated a concentration-dependent bactericidal activity with sterilization at $6-12 \mathrm{~h}$ at concentrations $>4 \times \mathrm{MIC}$, with a reduction in the bacterial count of $3 \log$ compared to the control in the first $6 \mathrm{~h}$ (fig. 2).

Owing to this data, complexes I and II may be classified as strong antimicrobial agents versus $S$. aureus and $S$. epidermidis strains. Their antimicrobial properties are very similar both in terms of potency and of the spectrum of sensitive strains. As complexes I and II only differ in the nature of the more labile ligand (the tetracetylthioglucose ligand in $\mathbf{I}$ and chloride in II) it is straightforward to assume that such ligand just plays an ancillary role in the overall antimicrobial activity while the triethylphosphine ligand constitutes an important part of the pharmacophore. The detailed mode of bacteria growth inhibition caused by these gold compounds will be the subject of further mechanistic studies; on the basis of existing literature, we can just state that there are two plausible mechanisms that warrant specific investigation, namely interference with selenium metabolism and inhibition of thioredoxin reductase and related enzymes (Jackson-Rosario Self 2009).

Overall, in view of the here reported in vitro results, it is evident that the clinically established antiarthritic drug auranofin demonstrates a high bactericidal activity against Staphylococcus sp; a similar antimicrobial potency is manifested versus the MRSA strains. Based on a preliminary set of experiments, the activity of auranofin appears to be characterized by a concentration-dependent character. These observations imply that AF is a promising agent that might be repurposed for the treatment of severe bacterial infections due to resistant Staphylococci; further investigations in animal models are needed. On the other hand, studies will be deepened to understand the effective molecular mechanisms through which auranofin manifests its potent antibacterial properties and to elucidate the reasons for the large differences in sensitivity between $S$. aureus and E. coli.

\section{Acknowledgements}

Beneficentia Stiftung and COST ACTION CM-1105 are gratefully acknowledged for generous financial support

\section{References}

Aroson JK (2007) Old drugs - new uses. Brit. J. Clin. Pharmaco. 64:563-565 Bondenson J (1997) The Mechanisms of Action of Disease-Modifying Antirheumatic Drugs: A Review with Emphasis on Macrophage Signal Transduction and the Induction of Proinflammatory Cytokines. Gen. Pharmacol. 29:127-150

Cavalla D (2013) Predictive methods in drug repurposing: gold mine or just a bigger haystack? Drug Discov. Today 18:523-532

Chong CR, Sullivan DJ (2007) New uses for old drugs. Nature 448:645-646

Clinical Laboratory Standards Institute (CLSI), National Committee for Clinical Laboratory Standard, Performance Standards for Antimicrobial Susceptibility Testing; Fourteenth International Supplement M100-S14, NCCLS, Wayne, PA, USA, (2004)

Craig WA (1998) Pharmacokinetic/Pharmacodynamic Parameters: Rationale for Antibacterial Dosing of Mice and Men. Clin. Infect. Dis. 26:1-10

Han S, Kim K, Kim H, Kwon J, Lee YH, Lee CK, Song Y, Lee, N Ha, K Kim (2008) Auranofin Inhibits Overproduction of Pro-Inflammatory Cytokines, Cyclooxygenase Expression and PGE2 Production in Macrophages. Arch. Pharm. Res. 31:67-74

Jackson-Rosario SE, Cowarte D, Myers A, Tarrien R, Rodney LL, Scott RA, Self WT (2009) Auranofin disrupts selenium metabolism in Clostridium difficile by forming a stable $\mathrm{Au}-\mathrm{Se}$ adduct. JBIC 14:507-510

Jackson-Rosario SE, Self WT (2009) Inhibition of Selenium Metabolism in the Oral Pathogen Treponema denticola. J. Bacteriol. 191: 4035-4040

Jackson-Rosario SE, Self WT (2010) Targeting selenium metabolism and selenoproteins: Novel avenues for drug discovery. Metallomics 2:112-116

Karampela I, Poulakou G, Dimopoulus G (2012) Community acquired methicillin resistant Staphylococcus aureus pneumonia: an update for the emergency and intensive care physician. Minerva Anestesiol. 78:930-940

Kuntz AN, Davioud-Charvet E, Sayed AA, Califf LL, Dessolin J, Arnér ES, Williams DL (2007) Thioredoxin glutathione reductase from Schistosoma mansoni: an essential parasite enzyme and a key drug target. PLOS Med. 4:1071-1086

Madeira JM, Gibson DL, Kean WF, Klegeris A (2012) The biological activity of auranofin: implications for novel treatment of diseases. Inflammopharmacology. 20:297-306

Messori L, Marcon G (2004) Gold complexes in the treatment of rheumatoid arthritis. Met. Ions Biol. Syst, 41:279-304.

Novelli F, Recine M, Sparatore F, Juliano C (1999) Gold(I) complexes as antimicrobial agents. Il Farmaco 54:232-236

Sanella AR, Casini A, Gabbiani C, Messori L, Bilia AR, Vincieri FF, Majori G, Severini C (2008). New uses for old drugs. Auranofin, a clinically established antiarthritic metallodrug, exhibits potent antimalarial effects in vitro: Mechanistic and pharmacological implications. FEBS Lett. 582:844847

Shaw F (1999) Gold-Based Therapeutic Agents. Chem. Rev. 99:2589-2600

Tejman-Yarden N, Miyamoto Y, Leitsch D, Santini J, Debnath A, Gut J, McKerrow JH, Reed SL, Eckmann L (2013) A reprofiled drug, auranofin, is effective against metronidazole-resistant Giardia lamblia. Antimicrob. Agents Chemother. 57:2029-2035

Zou J, Taylor P, Dornan J, Robinson SP, Walkinshaw MD, Sadler PJ (2000) First crystal structure of a medicinally relevant gold protein complex: unexpected binding of [Au(PEt3)]+ to histidine. Angew. Chem. 39:2931-2934 
